\title{
Power and Transformation in Higher Education Institutions: Challenges for Change
}

\author{
Peter Taylor and Susan Boser
}

\begin{abstract}
1 Introduction
The contributions to social change processes by universities have, in the past, been seen as 'diffuse and long-term'. Boothroyd and Fryer (2004) describe a long history of students acquiring knowledge and then going forth and applying it if they have the will and opportunity, while academics generate knowledge which others may use if they feel so inclined. Thus identified as consumers and producers of knowledge, respectively, there has not been a great deal of motivation for direct engagement by students and academics as actors in development and social change processes. Some countries do, of course, have strong histories of 'service learning', but this may still be carried out in such a way that the education institution distances itself from the change process itself. Where academic faculty have decided to commit themselves to real engagement with community development, they have often found themselves at odds with the requirements and expectations of the institution within which they are employed (Stoecker 2005). As universities are themselves shaped by wider society, the power relations that become embedded within higher education institutions may actually hamper and constrain the ways in which universities contribute to development and social change.
\end{abstract}

But this situation may be changing, with 'academics' and 'non-academics' working in partnership 'to analyse and solve social problems in a spirit of continuous mutual learning':

An alternative view of universities, now emerging, locates them more centrally and directly in the development process. Teachers, researchers and students are seen as development actors,

collaborating with others to help meet urgent social needs, and in the process enriching their own learning and that of the diverse people they work with. Community service by academics moves from the margins of the university, from being defined as a charitable donation of time over and above what academics really get paid to do, to become an integral part of intellectual discovery. In short, universities become socially engaged. (Boothroyd and Fryer 2004: 2)

In keeping with this growing trend, many individuals located in higher education institutions around the world have embraced participatory research in order to promote social change, and have sought actively to facilitate the learning of participation as a way of fostering transformation for social justice. Curiously, while many of us engage in such approaches in the field because of their capacity to redress power imbalances and contribute to wider social change, we often find ourselves blocked by particularly powerful individuals, groups or units, when we attempt to bring about or support processes of change within our own institutions. The asymmetrical power relations that create these blockages (whereby individuals or groups experience different ways in which they are constrained from action in a given situation) and a lack of understanding of why they occur, may prevent us from innovating or experimenting with alternative ways of working, such as expanding the use of participatory practices within our teaching or research, bringing about shifts in the culture of learning and teaching, or working in open and democratic ways with communities beyond the institution. They may hinder or even undermine the transformative learning that we hope our students will experience, and have a powerful influence on the way in which we shape and contribute to societal change.

In this article, and in keeping with the other articles in this IDS Bulletin, we seek to expand our 
understandings about the nature of power relations. Specifically, we attempt to unpack the constructions of power that constrain our actions and enable domination within higher education institutions, and explore how these may lead to blockages and constraints to change, learning and collaboration. We do this by drawing on cases of institutional change processes within two university settings, as well as stories and experiences shared by participants in an international dialogue on 'Learning and Teaching for Transformation'. To help with our analysis, we consider different approaches to understanding power and examine how these conceptual frameworks may lend themselves to efforts to affect change within our own institutions. We will explore whether reframing our understanding through the interplay of theory and practice might help to shift our focus from the 'one with power' to a network of socially determined boundaries, thus opening up a nuanced, complex field of possibilities for agency in which significant and positive change can occur.

\section{Stories of power and change in higher education institutions}

In January 2006, an e-forum dialogue took place on 'exploring the role of power in transformative processes' within the 'learning and teaching for transformation' (LTT) dialogue. The LTT initiative, with 300 participants from around the world, seeks to look critically at how professionals and practitioners, who are engaged in social change processes, learn (and are taught) within institutes of higher learning. It aims to deepen thinking about pedagogical approaches within higher learning that can challenge potentially influential people - from citizen and social movement activists to civil society and public sector leaders - to adopt participatory, inclusive and propoor decision-making processes in their work (Taylor et al. 2006).

Three key issues emerged through the rich dialogue of the e-forum that we feel are important for the theme of this IDS Bulletin. First, participants shared reflections on constructions of asymmetrical power relationships within their institutions. The views and ways of thinking about such power relations tended to be dichotomous, meaning located within a relationship with two sharply divided parts - one with the power, and one without, rather than a more nuanced and multidimensional vision of the context. For example:
Recently I am feeling of being blocked by dominant factors. I believe in training committed facilitators of change, but I am facing ethical dilemma. I am realizing that for facilitators to play their role there should be a minimum national level democracy, because facilitators face difficulties and may pay its price. (Bagher)

I view it more in the way the dominant group is able and willing to "allow" divergent views and involvement decision-making. How can dominant groups who thrive by exercising POWER OVER marginalised groups be persuaded to utilise more of power with or power within a group approach? (Abduba)

Several participants reflected on experiencing these assumptions as socially constructed:

The challenge I guess is not with power itself, but with the kind of relationship with which we use it, which I believe is more related to attitudes and mental models people and institutions develop and/or inherit. (Daniel)

... it seems to me that power is not just what you feel you have, but it is also importantly affected by how others perceive you and your position. But navigating the arenas of perceptions of power and the actual leveraging of power is a rather large grey area where one's own judgement, wisdom, sensitivity, etc. need to come into play. (Laurie)

A key question arises, therefore: When asymmetrical power relations exist within our own institutions, how do we transcend a dichotomous perspective and maintain awareness of the complexities and opportunities within that context?

Second, finding ways of seeing the world differently are not easy. 'Being' differently in the world, in the sense of praxis, whereby empowered individuals and groups engage with and apply theory as a tool to help shape the kind of work they want for themselves (see Introduction, this IDS Bulletin) is perhaps even harder. For individuals within higher education institutions to work collectively in such an endeavour requires mutual trust. As one of our colleagues remarked recently, 'there is a point when effective progress and change within our institution requires us ultimately to trust the university, trust the 
Dean, and trust each other'. Participants in the e-forum also made contributions on this issue:

The more a group can trust each other the better voice and bargaining power they have. Trust can facilitate to break "the culture of silence" as Freire mentions. Nonetheless, the macro hegemonic power structure can affect the micro trust building as well. Macro power structure often follows the policy of "divide and rule". Indeed, trust building process under undemocratic structure is slow, fragile and tedious. It is timeconsuming. It should be mentioned that sometimes the exercise of power to sabotage a building trust comes from someone within the project as well. Sometimes it is within the indigenous culture too. On the whole, I believe that if trust culture would be improved it could contribute significantly to the equalisation in power relations at micro and macro level. (Bagher)

But taking such an approach is not without challenges, and may incur risk:

I'm aware that I find it easy to think about or discuss power relationships when there is only a low chance of my being exposed to physical damage and/or life threatening power in my living and working environment. Were I to be faced with threatening power I know that would be different. It leads me to ask, how might I know courage? (Gerry)

Some called for confronting our fears:

... if we as individuals don't fear to take on us the burden of some more power (in addition to what others give us) we may stop undemocratic processes at early stages (Truls)

While others underscored that distrust may be a prudent and appropriate position:

As others have noted about their experiences, in many cases the "attitudes and mental models" that people have developed are ones of protectiveness, defensiveness and distrust ... I think that this distrust was quite useful given the context and the possibilities for exploitation and domination that cannot be denied. I think that their distrust was an important tool through which to leverage some mutual accountability and to maintain some sort of balance of power. (Laurie)

It can seem naive, even self-destructive, to behave in ways that are trustful, respectful and cooperative, and seek to encourage similar responses from others under such conditions. For example, we may believe that love and compassion are essential ingredients for our own well-being, and that our relationships with others should be founded on these tenets. But establishing such relationships, and even perhaps daring to mention them in institutional contexts where competition and asymmetrical power relations hold sway, may seem extraordinarily difficult for many individuals. Thus a second set of questions arose: Within adversarial contexts, how do we avoid responding with adversarial patterns ourselves? Under such circumstances, how do we find the courage to be true to our own values and practice attitudes, values and behaviours that bring mutual benefit? And how do we take care of ourselves, in engaging with others who respond in an adversarial manner? How do we sustain trust in ourselves and others when the path gets difficult?

A third key theme that emerged from the dialogue suggests that our own emotional responses may also play a role in inhibiting our efforts to transform institutions through participation. For example, internalised constructions about asymmetrical power relations may inhibit empowerment, in that perceptions of being in a 'power under' position can foster despair and hopelessness, undermining one's will to try to foster change.

... the use of power under adversarial relations inevitably leads to stalemate and frustration [and] blocks transformations. (Daniel)

Such constructions can also position us to react emotionally to the other's definition of the problem, without defining the vision or sets of possibilities for ourselves.

Besides, having an enemy is such an endless and tiring task and leads so quickly to despair! It never seems you are advancing as you base your victories on the "other" rather than your groups' well-

functioning and its creational-moments. (Kas)

Emotionally stressed reactions are not limited to adversarial conditions, however. Even when 
participatory values provide the grounding for all within a particular context, differences can still surface over understandings of what that means in practice, as can difference in priorities and capacities. Such conditions can produce stress and conflict that may strain our relationships with others. In light of this, a third set of questions emerged, namely:

How do we nurture ourselves and others during such times? What strategies enable us to maintain openness to learning during periods of conflict and stress? And what are the implications for the role of, and necessary skills for, leadership in participatory processes?

\section{Conceptual frameworks on power}

These three sets of questions are difficult to address, but in an attempt to explore them, we highlight here several conceptual themes that emerge with some diverging perspectives, and which have relevance for analysis of power in the higher education context. These involve: power as a function of a dyadic relationship as opposed to existing within a multidimensional milieu; power as capacity versus power as domination; power as external structure versus internalised self-limitations; and the concepts of habitus, capital and field (Bourdieu 1977).

\subsection{The dyadic relationship versus multidimensional context}

One of the key theorists on power over the last 30 years, Steven Lukes, views power as an instrument wielded by one party over another to influence the latter's actions, to prevent participation, or to shape the wants, desires and interests of the powerless (Lukes 2005). Yet others have argued that power is not limited to a dyadic relationship (that is, limited to only two actors - one with power and one without), but that it could exist in multidimensional ways, such as among multiple players and in complex roles, or embedded within structures. For example, Hayward (1998) rejects a dyadic construction of power as an action taken by a powerful party over a relatively powerless other party, in which power is both an instrument to be exercised and also a quality one either has or lacks. She notes that this construction requires some link between the two parties, whether immediate or distant. Hayward also challenges the notion of power as an instrument to be wielded, arguing it presupposes that, in the absence of the exercise of power, the powerless would be 'free' to choose an alternate action. She suggests, rather, that the powerless are not free in this manner because of the multiple social relations that impact on a sense of identity. Instead, Hayward conceptualises power as 'the network of social boundaries that delimit fields of possible action' (Hayward 1998: 9), with freedom being that which 'enables actors to participate effectively in shaping the boundaries that define for them the field of what is possible' (Hayward 1998: 12). This conceptualisation allows us to consider complex, patterned asymmetries among the ways in which individuals or groups are constrained from action in a given situation, without presupposing a dyadic relationship.

One particular strength of this model is its call for nuanced consideration of the constraints and possibilities for all actors, both those considered relatively 'powerless' and those considered relatively 'powerful'. It helps us to analyse the restraints on each stakeholder group stemming from their own political context, and promotes examination of the ways in which any social action constrains or enables actors in a given situation, thus resulting in shifts in asymmetries (Hayward 1998).

\subsection{Power as capacity vs power as domination}

Lukes distinguishes between power as capacity, or potentia, and power as domination, potestas. He views the latter as a sub-concept of the former and argues that power as dominance merits theorising and empirical research in and of itself. Lukes' particular focus is on the ways in which the powerful secure the compliance of those they dominate, with particular attention to the imposition of internal restraints (Lukes 2005).

Karlberg (2004) argues that focusing theoretical attention strongly on power in domination is too limiting. He suggests that power as capacity and power as domination are not exhaustive and mutually exclusive categories, and sees power as a capacity, neutral in itself, and with two critical dimensions. First, he advocates characterising the nature of the relationship between social agents as either adversarial or mutualistic. In adversarial power relations, two or more social agents seek to exercise power over another. Mutualistic power relations would reflect those in which two or more social agents are working in cooperation with each other. The second dimension refers to the balance of power between parties. Regardless of the nature of 
the relations, power, as capacity, may be balanced and equal among social agents, or it may be asymmetrical.

Thus, for example, one might find examples of asymmetrical, or unequal power that function well. Indeed, a hierarchical organisational structure, which involves differential power distribution, can function for the betterment of all when mutualistic relations exist. Or, in another case, adversarial relations can exist between social agents of equal power, resulting in a stalemate in which neither successfully dominates the other. Karlberg argues that the emphasis on understanding power in domination points toward a cultural tendency he calls normative adversarialism, or perspectives that see contest and conflict as the norm in social groups.

\subsection{Power as a externally structured vs internalised}

While Hayward does propose that internalised constructions about power play a role in social agents limiting their own range of decision making, her attention is drawn particularly to the structural and relational elements that inhibit choice. Similarly, Karlberg brings little attention to the role of the meaning individuals make of their experience. Lukes, on the other hand, brings a high level of attention to the matter of how willing compliance to domination is achieved. VeneKlasen and Miller (2002) explore this aspect of internalisation of power further, arguing that power can be visible (formal structures), hidden (exclusion and delegitimisation) and invisible (socialisation and control of information).

\subsection{Habitus, capital and field}

These different conceptual models raise a wide range of challenges and questions for our practice. For example, how do we integrate structural domination with individual capacities for human agency? And if, as Karlberg suggests, the dialogue and focus on power in domination, predisposes us toward adversarial relations, how do we achieve greater mutualism, trust and compassion, institutionally? How do we support and nourish mutual learning and collaboration, and care for ourselves and those with whom we work (colleagues, students, collaborators beyond our institution), abilities which seldom, if ever, appear in academic job descriptions. Valuable as the models described above are, they do not always take into account the role played by our own constructions about power, specifically that the internalisation of a dyadic construction of power may indeed be one way in which domination persists.

Bourdieu (whose theories are discussed in greater detail by Navarro and by Moncrieffe, this IDS Bulletin), recognises education as a particularly significant mechanism for the legitimating of 'doxa' subsystems of society with rules and purposes of their own, each accommodating a set of tools, knowledges and skills (Taylor and Fransman 2004). Linked to the doxa is the 'habitus', an internalised disposition or trained capacities and structured propensities to think, feel and act in determinate ways, which then guide people in their creative responses to the constraints and solicitations of a particular cultural milieu (Wacquant 2004).

Within higher education institutions, Bourdieu helps us perceive how paralysis and hopelessness may set in, encouraged by a sense of futility that accompanies our dyadic constructions of power relations, viewing the dominant as monolithic and impenetrable. The asymmetries in power relations experienced in society are reproduced socially within institutions that are derived from this society, all with the purpose of acquiring different forms of 'capital'. Cultural capital in particular may include academic qualifications and symbolic capital, including the legitimacy conveyed by membership of academic institutions.

Bourdieu also introduces the notion of 'field', defined as a network of relations among positions that can be objectively defined in terms of their capacity to advance their own self-interest as well as by their relation to other positions (Bourdieu and Wacquant 1992). For Bourdieu, fields, particularly in complex social groups such as bureaucracies or even advanced societies, are not constituted by dyadic dominators and subordinates. Rather they are fluid and complex, comprised of multiple interests, and distinct yet interconnected, affecting each other.

Applying this concept to our understanding of power relations in education institutions can help us break down the dyadic constructions of 'us' versus a monolithic, impenetrable 'them' that paralyse and block action. The concept of field enables us to analyse the context as complex and multidimensional, with multiple constituents who bring varied interests, and who each exist within other fields that both support and restrain their 
action. We can also view these fields as dynamic, fluid, and therefore amenable to change. This has implications for action in that, by application, we can identify the spaces for strategic engagement and interaction within the context. We may also identify the common interests that exist among the constituents (academic staff, students, etc.) and use these as the foundation for dialogue (Fisher and Ury 1991). We can consider ways in which we might influence the structures themselves, perhaps by including new constituents or altering the patterns of interaction and communication among existing constituents, thereby reconstituting the fields.

We may even argue that altering the fields themselves through greater participation has the potential to similarly alter the habitus for oneself and others. Several participants in the LTT dialogue shared instances where the process of interaction itself within a participatory process played a key role in changing constructions and exercise of power. Coming to know the other through social interaction and, through that, developing trust, contributed to mutualistic relations and opened up possibilities for altering power relations. For example:

... the "process" of getting people to even think about participating ... is far more important than the programme content. It is the internalised "process" of deciding whether or not to meet, then maybe engage in educational dialogue with "the oppressor" that creates empowerment. Then, if people do agree to participate, it tends to be the "social interaction" that creates self empowerment, not the course content. (Marcus)

When looking back on the past, more than victories or defeats what rather comes to mind is certain people who have marked me. The most powerful folks l've worked with had a mixture of modesty, constancy and dignifying determination and knew that to create an independent, constructive power, rooted in the group/movement itself was relevant to achieve the outcomes. Passionate participation, transparency in each step, complicity, tone of voice, limiting long interventions in meetings or even too many meetings ... was essential. Publishing not only claims, but also the positive results, no matter how little they were. Giving thanks, giving thanks for every little thing to every single person, especially if being outsiders/ "enemies". (Kas)

\section{Linking concepts to action - tools for change}

We have suggested here that conceptual understanding is vital if we are to throw light on the ways we understand and think about power relations in our higher education institutions. We need, however, to identify also practical actions which can help bring about, or contribute to, processes of positive change. How may we ensure that the ways in which power is played out, shaped largely by the society in which we are located, does not impede the potential for transformative learning among our colleagues, our students and others with whom we collaborate and learn through collective action? How can we avoid this inhibiting them from engaging in further processes of development and social change? We need guidance and tools for change that can lead us and enable us to take action to alter the status quo; these tools may lie in the intersection of participatory practices within our own institutions, with the conscious application of theory aimed at counteracting internalized constructions of asymmetrical power relations.

Bourdieu's (1990, 1977) notions of 'habitus', 'capital' and 'field' have huge potential for us by enabling us to re-imagine the complex web of power relations we encounter and indeed propagate within our institutions, but his contribution to our discussion extends further through the possibility of tools that help us translate our theoretical understandings into action.

The first, 'reflective practice', a key element of participatory research approaches (and discussed extensively by Pettit in this IDS Bulletin) provides us with opportunities to access and explore different forms of knowledge through critical reflection on our experience, thus deepening our learning. Bourdieu introduces us to the concept of 'reflexive analysis', emphasising our need to critique our own locations and our own habitus. 'Reflexive analysis, which teaches that we are the ones who endow the situation with part of the potency it has over us, allows us to alter our perception of the situation and thereby our reaction to it' (Bourdieu and Wacquant 1992: 136.) He suggests here that internalised constructions about relations of power do indeed affect our definitions of situations, as well as our actions. He offers reflexive analysis as one tool to expose such constructions, freeing us to explore new alternatives.

Through reflexive analysis, we may also become aware of how others perceive us in terms of power relations. 
As members of higher education institutions, we acquire status and acknowledgement in many societies, which brings privilege as well. We are part of a recognised academic community, and so we may find relations with those outside the academic context or even with others within our institutions, affected by such perceptions of privilege. Might others have constructions about power relations involving us that influence their perceptions about their own choices? Reflection on our relationships with others may suggest ways in which we can create spaces for education and awareness. For example:

When I think about power that resulted from group processes ... Most often power to take action was revealed in a different form than I expected. What I thought I needed to do to move a situation changed. (Candee)

Reflexive analysis also enables us to consider linguistic relations. 'Linguistic relations are always relations of symbolic power through which relations of force between the speakers and their respective groups are actualized in a transfigured form' (Bourdieu and Wacquant 1992: 142). Bourdieu states that we cannot fully appreciate language without understanding the field and habitus of the context. Can language then also help us to decipher the relations of power? Can it be used to support reflexivity? And if so, can language also be used to alter relations of power? As an LTT e-forum participant commented:

... an important aspect of power is who is at the table and who can be at the table. In other words, the nature and the architecture of the forums that we choose for participation and representation including, of course, language. (Nanci)

We believe that reflexive analysis on the language we use, with attention to ways in which we refer to others, to the structure and to ourselves that highlights the problems, the limitations, the strengths or the progress that we are making, can foster hope, an openness to learning and a sense of our own power.

\section{Learning from experiences of change}

Appreciating that critical reflection on one's own experience within a particular social context is a key step to understanding, and thus potentially altering, relations of power, in the remaining part of this article we reflect on two change processes, in which we have been engaged personally. These allow us to explore asymmetries in power relations, and to see how the different concepts and theories discussed above may play out in practice. Both cases were informed by the exchange within the LTT dialogue over several years, by our own theoretical understandings of power, learning and education, and also through our respective experiences as educators and practitioners in a range of different contexts.

\subsection{Case 1: Assessment, power and change in a US college}

Throughout 2005 and 2006, I (S.B.) led a project within my college that illustrates application of these concepts and strategies. This example is located in the context of the USA, where higher education institutions are being required increasingly by accrediting bodies to assess student learning. Over the past three years, one accrediting body had required the College of Education at this university to design and implement such assessment in all courses that might be taken by education majors. Faculty in many departments in other colleges expressed strong resistance to assessment as was mandated. While plans were put in place in all departments, many faculty members demonstrated passive resistance to developing quality measures, participating and entering data, or using the findings for the department. Further, just as this assessment system was being fully implemented and resistance was reaching a peak, the main accrediting body for the university as a whole mandated that assessment for all departments and programmes needed to be in place within the next few years.

I was asked by the Dean to lead a small committee of department representatives, who were charged with the task of implementing assessment within the College of Humanities and Social Sciences. At that time, I was a junior, faculty member without the security of a permanent appointment (tenure), a position of relative vulnerability and lack of power within the university academic hierarchy.

However, as the Dean and I both shared a commitment to participatory processes and supporting faculty voice, the process began with listening closely to the definition of the problem as seen through the eyes of the faculty. The faculty clearly expressed the feeling that they were trapped within a dyadic relation of power with the dominant 
College of Education; further, they saw the upcoming assessment of the larger accrediting body as being only more of the same. They felt powerless to stop or alter this process, which they saw as requiring time-consuming tasks that had no meaning for them, yet significantly added to their already strained workloads.

Drawing on both tools of participation, Bourdieu's theory, and insights gained through the LTT e-dialogue on power, I used the following strategies for facilitating this process:

- Attending carefully to creating conditions that engendered trust and nurtured the involved faculty, using tools such as: listening closely to their definition of the situation; affirming faculty experience and feelings, and seeking to clarify the implications these concerns had in the larger context; identifying the faculty definition of the problem and developing a workplan that responded to that; careful and transparent communication with the committee and with the college as a whole on this process; identification and affirmation of the positive steps and successes; consistently following up on agreedupon actions.

- Engaging in reflexive analysis about the beliefs regarding the relations of power committee members experienced within their own departments. This resulted in several strategies to understand and reconstitute these fields. These included expanding the committee to include those who were higher in the hierarchy within the departments; meetings with the department chairs; holding a public debate to give multiple perspectives a chance to be heard; holding open, public short discussion meetings to give all the faculty direct access to air their concerns.

- Applying analysis of the field of the larger environment, the committee conducted some research that included: (1) the regulatory environment that the College of Education was in, enabling the committee to better understand their particular needs and interests, and craft a counter-proposal for assessment strategies that met that College's needs, while also meeting the departments' needs, and (2) the allied interests within the university and potential opportunities for altering the current mandates.
- Listening closely to the language that was used by the various parties in order to identify and address points of miscommunication.

- Engaging in periodic reflection on what was working well for us and what had we learned, and modifying our workplan and strategies accordingly.

One key revelation was that many of the faculty (though not all) were not averse to assessment itself; indeed, many were quite dedicated to teaching and their programmes and, as social scientists, were interested in developing tools that could help them learn about the impact of their work and how they might grow. The challenge was that assessment processes had become conflated with the College of Education's rather directive process of implementation. Not only did that approach engender resistance, but the ways in which power was exercised to force assessment resulted in assessment that did not meet other faculty needs and was costly to implement.

This process has seen many positive outcomes, including a significantly increased voice for these departments and the college as a whole in developing assessment that is owned and used by the faculty; an increase in resources to support assessment processes and more equitable relations with the College of Education.

\subsection{Case 2: Curriculum reform within a process of strategic change in a UK institution}

At the Institute of Development Studies (IDS) in the UK, a process of curriculum development was instigated in which a key element of the postgraduate (Masters) teaching programme would be revised. This process, in which I (P.T.) acted as the facilitator by remit of my responsibility for the teaching programme at the institute, was informed by a detailed review undertaken of the teaching programme and through extensive feedback provided by staff, students, external examiners, university colleagues, alumni and other interested parties. Guidelines were consulted from external, national sources and findings were utilised from a wider framework of education-related research, including the LTT dialogue discussed earlier in this article.

The curriculum change process was situated within a wider process of strategic change at IDS, 
implemented soon after the arrival of a new Director, with a strong emphasis on the successful integration of research, teaching and knowledge services. Teaching was seen as a key element of the Institute's strategy, and apart from the need to revise the teaching programme to meet national education guidelines, there was a wider intention of addressing the need for greater coherence, accountability and efficiency within a quality development studies education programme.

A series of meetings was organised, to which staff and students of IDS were invited, as were members of other university bodies and departments. $A$ proposal for initiating change in the teaching programme was also submitted to the IDS management group. After lengthy debate in these different fora, a series of aims were identified, which articulated the need for IDS to move beyond current perceptions of the objectives of the teaching programme. This led to agreement on a widened goal of expansion and further specialisation of the teaching programme, and also that of increasing overall student numbers.

A working group was formed on the basis of responses to an invitation to institute staff engaged in teaching, and a process of curriculum development began in earnest. At this point, a trajectory of struggle and resistance was observed. The ensuing events demonstrated that a dyadic framing of power was a completely inadequate explanation for the fact that at different times, different groups and individuals either supported or resisted change. On occasions, the process appeared to move rapidly with what appeared to be logical ways forward being identified, only for these recommendations to be seen as a source of huge contention due to the perceived effect they would have on existing Masters programmes. While the broad goals, and an abstract vision of a changed teaching programme were seen as unthreatening, the move towards detailed proposals which would bring about real change elicited reactions that could potentially undermine the entire process.

Resistance was typified by heightened exchanges of emails, rumours and alternative rendering of decisions and rationale, accompanied by suggestions that the actual process had lost its legitimacy by failing to offer options for wider consultation at specific stages. Certain key individuals did not participate at critical moments when the reasoning for proposals was explored, and so did not have the full background to help them understand why particular avenues were being followed. As a result, communication became confused and circular arguments ensued. I found myself in an embattled position on numerous occasions, receiving strong support from some quarters, and considerable opposition from others. Sometimes, those who offered support then appeared to ally with those resisting, and vice versa.

Why was the process so difficult? Returning to the concepts considered earlier in this article, we could conclude that it was indeed necessary to understand the power asymmetries being observed in order to find a way forward and to achieve the goal which had not seemed so difficult to reach prior to actual change beginning. Following Bourdieu, we might consider that the habitus of the IDS social world ensured strong dispositions to resist the idea of change in certain areas where capital might be affected, and an inertia existed which would slow or prevent such change taking place. Cultural capital was invested in the existing academic qualifications of the teaching programme, and the idea of actually bringing about change in these, even to the point of reconfiguring the programmes such that existing qualifications might be reframed within a more integrated structure, was considered by some to be too great a shift. Interestingly, the trajectory of resistance played out at two levels, first within IDS itself and second, more widely, in the University, once formal proposals were made at a higher level in the institutional structure.

Unlikely as it sometimes seemed along this difficult road, actual change did finally take place. In ways rather similar to those utilised by S.B., I attempted to build a sense of trust and responsiveness to concerns. Alliances were strengthened not only with those who were supportive but with those who had reservations about the changes taking place. Individuals who had shown resistance were encouraged to participate more fully in discussions and debate. Confusion over the nature of key discussions was addressed by providing further and fuller information and explanations, sometimes requiring further research to respond to legitimate questions. Rather than seeking refuge in an adversarial stance, I attempted to provide leadership through a mutualistic and appreciative approach with 
all those concerned, including staff and students. Reflective moments were shared with colleagues on what was being experienced, in an effort to find constructive ways of engaging with all parties. At times, these reflections revealed assumptions, including my own, that had been made along the way and which may have contributed to misunderstandings and gaps in knowledge of what was taking place. Attention was given to language, in an effort to avoid expressions of blame and anger. Flexibility and willingness to accommodate genuine concerns was seen as essential contributors to the process moving forward. Perhaps most importantly, I experienced moments of real care and compassion from colleagues when challenges appeared to become overwhelming. The renewed energy derived from these crucial moments hopefully was modelled again in relationships with others engaged in the ongoing dialogue. All of these factors combined, we imagine, to create an opportunity for real change to take place, bringing benefits ultimately for members throughout the institutional community.

\section{Concluding reflections}

What can be learned from the experiences and cases shared above? We believe we can draw on these to help us see a few possibilities for bringing about positive change processes within higher education institutions. For example, our experience suggests that the use of participatory processes can indeed shift group culture from the adversarial to one that is mutualistic. By influencing perspectives and practice within our immediate context, might some participants also alter their own practice in other venues? An understanding of power as existing in multidimensional ways, among multiple players and complex roles might offer hope that such influence can indeed be felt in ways that are beyond our immediate view. In those instances in which mistrust is largely a function of our constructions, participatory practice, openness to learning and holding our own constructions lightly might enable us to identify and engage pockets of support among those placed higher in the hierarchy that we might not otherwise recognise.

Another key learning for us relates to the ways in which teachers in higher education institutions can themselves learn about power relations by reflecting on their own experiences of institutional change, and may draw on this to inform the way in which they relate to students. As we noted in the introduction, universities often are extremely hierarchical places, where staff find themselves governed by either seen or unseen power structures, and students find themselves at 'the bottom of the food chain'. For development studies' students, as typified in the second case study, their educative experience may well help to determine the way in which they relate to others once they embark or continue on their professional life, acknowledging of course that much of their behaviour is determined by earlier upbringing and life experience. For education to be a truly transformative learning experience, the interplay between theory and practice within universities must be such that it contributes to shifts in unequal power relations experienced in society and reproduced within the institution itself. By reshaping asymmetrical power relations in this way, and by encouraging learning conditions that are supportive, caring, stimulating and in touch with forms of knowledge that include, yet go beyond, the cognitive and intellectual, we feel there is a real possibility to prepare and empower students to make a positive contribution to development and social change.

So, although such perspectives present challenges for us, they provide an opportunity for us to reimagine ourselves in relation to others; to take a fresh view at the way in which we conceive our own power and the power of others; to reconsider how we nurture and sustain our own well-being, while building and nourishing constructive discourse with others both within and outside our institutional contexts. We believe it is only through a constant revisiting of theory in relation to our own practice, and a sharing of our reflections on actions with others in our field that we have an opportunity to bring about wider institutional learning and change in the context of higher education. By encouraging and supporting greater reflexivity in institutions, including those we have described in the case studies above, we believe that academic institutions have a real opportunity to not only change themselves, but to support development and social change more widely through collaboration and partnership that draws on a genuine understanding of power relations, and is observed through positive, practical action for change. 


\section{References}

Boothroyd, P. and Fryer M. (2004) 'Mainstreaming Social Engagement in Higher Education: Benefits, Challenges and Successes', paper presented at the UNESCO Forum Colloquium on Research and Higher Education Policy, Paris, 1-3 December

Bourdieu, P. (1990) In Other Words: Essays Towards a Reflexive Sociology, Stanford: Stanford University Press

Bourdieu, P. (1977) Outline of a Theory of Practice, Cambridge: Cambridge University Press

Bourdieu, P. and Wacquant, L.J.D. (1992) An Invitation to Reflexive Sociology. Chicago: University of Chicago Press

Fisher, R. and Ury, W.L. with Patton, B. (eds) (1991) Getting to Yes: Negotiating Agreement Without Giving In, 2nd edn. New York: Penguin Books

Hayward, C.R. (1998) 'De-facing Power', Polity 31.1: 1-22 Karlberg, M. (2004) Beyond the Culture of Contest, Oxford: George Ronald Publisher

Lukes, S. (2005) Power: A Radical View, 2nd edn. Hampshire: Palgrave Macmillan
Stoecker, R. (2005) Research Methods for Community Change. A Project-based Approach, Thousand Oaks: Sage

Taylor, P., Pettit, J. and Stackpool-Moore L. (2006) 'Learning and Teaching for Transformation Insights from a Collaborative Learning Initiative', in P. Gurstein and N. Angeles (eds), Learning Civil Societies: Shifting Contexts for Democratic Planning and Governance, Toronto: University of Toronto Press

Taylor, P. and Fransman, J. (2004) Learning and Teaching Participation: Exploring the Role of Higher Learning Institutions as Agents of Development and Social Change, IDS Working Paper 219, Brighton: IDS

VeneKlasen, L. and Miller, V. (2002) A New Weave of Power, People \& Politics: the Action Guide for Advocacy and Citizen Participation, Oklahoma City: World Neighbors

Wacquant, L.J.D. (2004) 'Critical Thought as Solvent of Doxa', Constellations 11.1: 97-101 